\title{
Coupled Effects of Strain Rate and Temperature on Deformation Twinning in $\mathrm{Cu}-\mathrm{Zn}$ Alloy
}

\author{
P. Zhou, ${ }^{\text {a }}$ J. Q. Zhou, ${ }^{\text {abb, } 1}$ Z. X. Ye, ${ }^{a}$ E. Jiang, ${ }^{a}$ W. B. Hu, ${ }^{a}$ and H. L. Le ${ }^{a}$ \\ ${ }^{a}$ School of Mechanical and Electrical Engineering, Wuhan Institute of Technology, Wuhan, Hubei, \\ China \\ ${ }^{\mathrm{b}}$ School of Mechanical and Power Engineering, Nanjing University of Technology, Nanjing, Jiangsu, \\ China \\ ${ }^{1}$ yyzjqce@sohu.com
}

$\mathrm{Cu}$-Zn alloy is an advanced material, but its deformation twinning mechanism still keeps unknown so far, especially the couple effects of temperature and strain rate. In this paper, a theoretical model of $\mathrm{Cu}-\mathrm{Zn}$ alloy is proposed by considering the coupled effects of strain rate and temperature. The model can predict the experimentally observed tendency of the spacing evolution of twin boundary (TB) accurately, and it is known that low temperature and high strain rate will promote deformation twinning. Moreover, deformation twining is more susceptible to low temperature than to high strain rate, and TB spacing and twin layer thickness will decrease with high strain rate and low temperature.

Keywords: $\mathrm{Cu}-\mathrm{Zn}$ alloy, deformation twinning, strain rate, temperature.

Introduction. $\mathrm{Cu}-\mathrm{Zn}$ alloy is an advanced material, but its deformation twinning mechanism still keeps unknown so far, especially the couple effects of temperature and strain rate. Deformation twinning has been investigated in many materials as an important plastic deformation mode because of its special deformation mechanism [1-4]. The strengthening effect of coherent twin boundary can hinder the motion of dislocations to improve the intension of the material in accordance by grain boundary [5]. Recently, the impact strength of nanoscale twins can be up to $1 \mathrm{GPa}$ when each grain is introduced by a high density of coherent growth TBs [6]. Meanwhile, the maximum strength will appear when the twin-boundary space reaches $\lambda=15 \mathrm{~nm}$, and the strength will decrease when $\lambda$ continues to shrink less than $15 \mathrm{~nm}$ [7]. The factors, such as strain rate and temperature, are considered to affect deformation twinning [5-8], and it is widely known that high strain rate and low temperature can lead the growth of the twins. The effect of strain rate and temperature on deformation twinning in coarse-grained fcc metals and alloys had been summarized by Christian and Mahajan [1]. The observation of CG-Cu (coarse crystal-Cu) twins will not appear moderating strain level at a very high strain rate or low temperature under RT quasi-static deformation, which has been shown in many experiments [8-11]. Based on the above investigations, we can see that the temperature and strain rate can promote twinning, which has a great influence on the property of a certain material. However, the coupled effect of strain rate and temperature on deformation twinning in fcc materials is still unknown so far. Twin boundaries and grain boundaries improve the flow stress of the materials, and the flow stress will increase with the decrease of TB space before reaching a critical size. Twin growth seems like a insert process when the temperature and strain rate is range [12]. This paper aims to build a theoretical model for $\mathrm{Cu}-\mathrm{Zn}$ alloy, which determines how the deformation twins (DT) are generated by tensile deformation and how they grow with the change of strain rate and temperature. In addition, the theoretical model will analyze the role of DTs on the mechanical behaviors of the material. 


\section{Theoretical Model.}

1.1. Deformation Mechanism of Cu70-Zn30. Slip and twinning are the major modes for metal-plastic deformation, and it is closely related to the stacking fault energy (SFE) of a certain material. In $\mathrm{Cu}-\mathrm{Zn}$ alloy, when the content of $\mathrm{Zn}$ increases gradually from 0 to $30 \%$, the SFE of the alloy will decrease gradually from 80 to $14 \mathrm{~mJ} / \mathrm{m}^{2}$ [13]. In this research, Cu70-Zn30 which owns a low SFE is easy to form a stacking fault. Meanwhile, the material owns a large number of twins at room temperature, and the twinning deformation is the main deformation mechanism of $\mathrm{Cu} 70-\mathrm{Zn} 30$.

1.2. The Flow Stress of $\mathrm{Cu} 70-Z n 30$. The flow stress of the metal mainly attributed to impeding of internal barriers to the motion of dislocations. According to the scope of the obstacle, the flow stress is divided into short-range and long-range stress. The short-range stress can be modified by thermal activation movement, and it will increase when temperature declines or strain rate increases. The barriers of long-range stress contain grain boundaries, twin boundaries and other microstructure which mainly relates to strain [14].

The flow stress of the material $\sigma$ can be expressed as

$$
\sigma(\varepsilon, \dot{\varepsilon}, T)=\sigma^{*}(\varepsilon, \dot{\varepsilon}, T)+\sigma_{a},
$$

where $\sigma_{a}$ is the long-range stress (thermal stress) of the resistance to the dislocation motion and $\sigma^{*}$ is the short-range stress (activation stress). Due to thermal activation stress is affected by temperature strain and strain rate, Johnson-Cook equation is adopted [15].

$$
\sigma=\left[\sigma_{0}+B \varepsilon^{n}\right]\left[1+C \ln \varepsilon^{*}\right]\left[1-T^{*}\right]^{m},
$$

where $\varepsilon$ is the strain, $T^{*}=\frac{T-T_{r}}{T_{m}-T_{r}}, T$ is the absolute temperature, $T_{m}$ is melting temperature of the material, $T_{r}$ is the reference temperature taking the value of $77 \mathrm{~K}$ in this paper, $\varepsilon^{*}=\dot{\varepsilon} / \dot{\varepsilon}_{0}$, where the reference strain rate $\dot{\varepsilon}_{0}$ is $0.001 \mathrm{~s}^{-1}$, and $\sigma_{0}, B$ and $n$ are the material constants; $C$ and $m$ represent the coefficient of strain rate hardening and thermal softening exponent.

The thermal stress will adopt the power strengthening law in [14]. The long-range stress $\tau_{a}$ can be taken as a function of the plastic strain, that is

$$
\tau_{a}=\tau_{a}^{0} \gamma^{n_{1}}
$$

There are no related experiments to give the values of $\tau_{0}^{a}$ and $n_{1}$, setting $\tau_{0}^{a}=380 \mathrm{MPa}$ and $n_{1}=0.4$, which refers to the tensile unloading experiment about OFHC $\mathrm{Cu}$ (oxygenfree high conductivity copper) [14] and CG-Cu tensile test [16]. Using the relationship between the normal and shear stress through the Taylor factor $M$, that is $\sigma_{a}=M \tau_{a}$, $\gamma=M \varepsilon$ :

$$
\sigma_{a}=M^{1+n_{1}} \tau_{0}^{a} \varepsilon^{n_{1}}
$$

The flow stress combined with Eqs. (2) and (4) can be expressed as

$$
\sigma(\varepsilon, \dot{\varepsilon}, T)=\left[\sigma_{0}+B \varepsilon^{n}\right]\left[1+C \ln \frac{\dot{\varepsilon}}{\dot{\varepsilon}_{0}}\right]\left[1-\left(\frac{T-T_{r}}{T_{m}-T_{r}}\right)^{m}\right]+M^{1+n_{1}} \tau_{0}^{a} \varepsilon^{n_{1}} .
$$


1.3. The Local Stress and the Strain Hardening Role of Deformation Twins. The plastic deformation mechanism is related to the SFE value of a certain material. $\mathrm{Cu} 70-\mathrm{Zn} 30$ has a large sum of deformation twins at room temperature, so the major plastic deformation mechanism is deformation twinning. Twin boundary can hinder the motion of dislocations. With the purpose of investigating the evolution of deformation twinning at different deformation conditions, it is necessary to describe the diagram of schematic structure about twins. Twin lamellae and matrix lamellae (twin space) which are divided by crystallographic plane form a three-dimensional twin grain. In this paper, the thickness of twin layer is defined as and the neighboring TB spacing is defined as $\lambda$.

1.3.1. Local Stress. Basis for Taylor dislocation hardening model [17], for fcc materials, the stress is caused by dislocation increases with the increasing of dislocation density $\rho$,

$$
\sigma_{0}=M \alpha G b \sqrt{\rho}
$$

where $M$ is the Taylor orientation factor, for fcc metals $M=3.06, b$ is the Burgers vector magnitude, $\alpha$ is the empirical material constant, and $G$ is the shear modulus. For equally distributed dislocation, the average spacing $\Delta L$ between the two connected barriers and the dislocation density $\rho$ are satisfied with the equation $\Delta L \approx \rho^{-1 / 2}$, will be instead of in this paper, therefore

$$
\sigma_{0}=M \alpha G b / \lambda
$$

1.3.2. The Strain Hardening Role of DTs. The DTs which have a strain hardening mechanism are effected by the flow stress and the strain. The twin boundary and grain boundary can hinder the movement of dislocation, also, the grain size can improve the strain hardening. The schematic illustration of strain hardening about DTs is shown in Fig. 1.

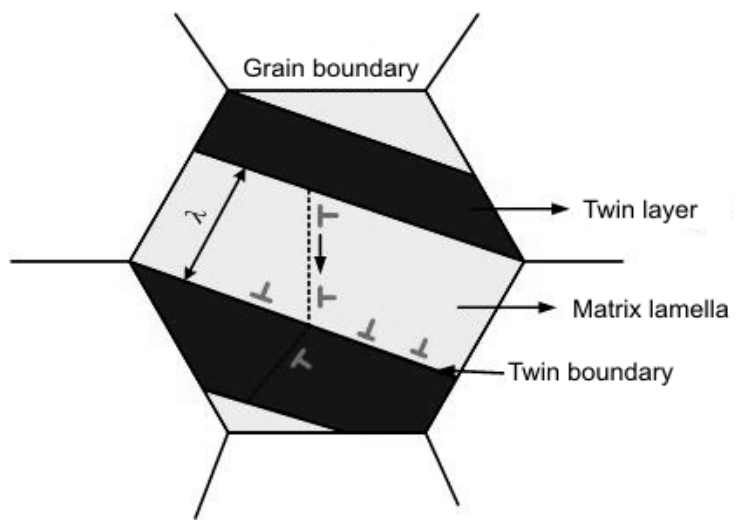

Fig. 1. Schematic illustration of strain hardening about DTs.

The formula of the strain hardening about DTs can be written in

$$
\Delta \sigma=K_{T} \lambda^{-1 / 2}
$$

where $K_{T}$ is $\mathrm{H}-\mathrm{P}$ slope for twinning and $\lambda$ is the average distance between the neighboring twin layers (TB spacing).

The total flow stress also can be obtained by combining Eqs. (7) and (8), that is

$$
\sigma=M \alpha G b / \lambda+K_{T} \lambda^{-1 / 2} .
$$


This leads to

$$
M \alpha G b / \lambda+K_{T} \lambda^{-1 / 2}=\left[\sigma_{0}+B \varepsilon^{n}\right]\left[1+c \ln \frac{\dot{\varepsilon}}{\dot{\varepsilon}_{0}}\right]\left[1-\left(\frac{T-T_{r}}{T_{m}-T_{r}}\right)^{m}\right]+M^{1+n_{1}} \tau_{0}^{a} \varepsilon^{n_{1}}
$$

\section{Results and Discussion.}

2.1. Comparisons with Experimental Results. In this study, we carried the numerical simulation and the constitutive model is validated by the results. The results about twin spacing evolution under uniaxial compression were taken from Xiao et al. [16]. The samples were subjected to dynamic plastic deformation at a liquid nitrogen temperature (LNT-DPD). The corresponding prediction by the theoretical model is plotted in Fig. 2 together with the extracted experimental data. The parameters is used to calculate Eq. (10) for Cu70-Zn30 which are summarized in Table 1. It can be seen that the predicted results about twin spacing evolution under uniaxial tensile are suitable for experimental points. The twin spacing will decrease when strain increases. The strain is defined as $\varepsilon=\ln \left(L_{0} / L_{f}\right)$, in which $L_{0}$ and $L_{f}$ are the original and final thickness of the deformed sample.

$\mathrm{T}$ a b 1 e 1

Parameters Used in Calculation in Eq. (10)

\begin{tabular}{|l|c|c|c||}
\hline \multicolumn{1}{|c|}{ Parameter } & Notation & Value & Reference \\
\hline H-P slope for twinning & $K_{T}$ & $0.31 \mathrm{MN} / \mathrm{m}^{3 / 2}$ & {$[18]$} \\
\hline Empirical material constant & $\alpha$ & $0.2-0.5$ & {$[19]$} \\
\hline Burgers vector & $b$ & $0.256 \mathrm{~nm}$ & {$[20]$} \\
\hline Taylor orientation factor for fcc metal & $M$ & 3.06 & {$[21]$} \\
\hline Shear modulus & $G$ & $37 \mathrm{GPa}$ & {$[20]$} \\
\hline Yield stress & $\sigma_{0}$ & $112 \mathrm{MPa}$ & {$[15]$} \\
\hline Strain hardening exponent & $n$ & 0.42 & {$[15]$} \\
\hline Coefficient of strain hardening & $B$ & $505 \mathrm{MPa}$ & {$[15]$} \\
\hline Coefficient of strain rate hardening & $C$ & 0.009 & {$[15]$} \\
\hline Melting temperature & $T_{m}$ & $1189 \mathrm{~K}$ & {$[15]$} \\
\hline Thermal softening exponent & $m$ & 1.68 & {$[15]$} \\
\hline
\end{tabular}

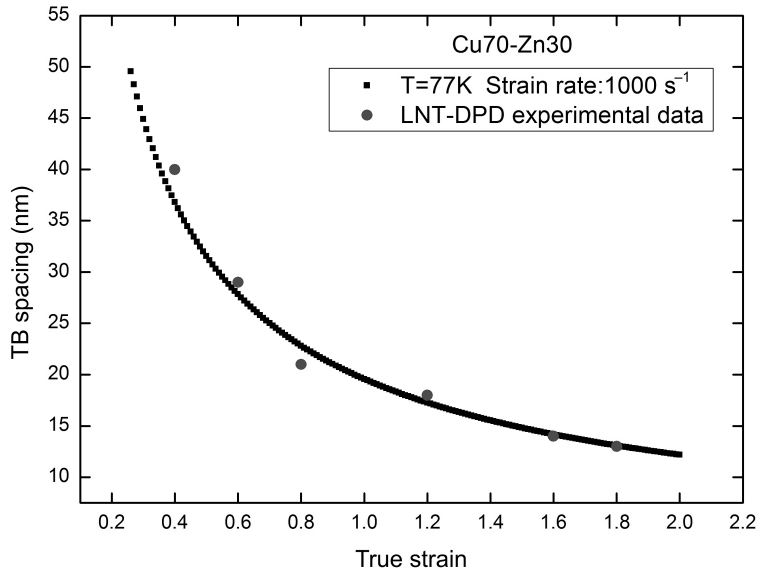

Fig. 2. Comparison between the calculated and experimental TB spacing-strain curve under dynamic plastic deformation at a liquid nitrogen temperature. 
2.2. The Evolution of Twin Layer Thickness. According to the relationship between TB spacing $\lambda$ and average twin lamellae thickness $t$ by Karaman et al. [22]:

$$
\frac{1}{\lambda}=\frac{1}{2 t} \frac{f}{1-f}
$$

where $f$ is the volume fraction of twinned region. Further, we can get:

$$
t=\frac{\lambda}{2} \frac{1}{\frac{1}{f}-1} .
$$

It is well known that low temperature and high strain rate can promote deformation twinning. The value of $f$ will rises when temperature shrinks and strain rate increases. So the thickness of twin layer will decrease when TB spacing decreases. In the history of deformation, dislocations are hindered by TBs. When the deformation process continues, dislocations start to pile up and produce a stress concentration. With strain increasing, dislocation accumulation in the twin boundary leads to stress concentration. The magnitude of stress concentration is affected by the dislocation accumulation. As the twin spacing $\lambda$ and twin layer $t$ decreases with increasing strain, fewer dislocations are expected to pile up. It means a higher external stress is required for the dislocations to cross TBs, so smaller thickness of twin layer and TB spacing make a significant contribution to the flow stress of fcc metal.

2.3. Temperature Effect of Deformation Twinning. Figure 3 shows the dependence of TB spacing evolution on the temperature at different strain rates 1000 and $0.001 \mathrm{~s}^{-1}$. As we can see, the TB spacing $\lambda$ decrease first with the decreasing of $T$ within the range between 77 and $1096 \mathrm{~K}$. It can be attributed to the suppression of dislocations thermal activation at a low temperature. Under low temperature, the dislocation motion is restrained and the twinning becomes the dominant mechanism of plastic deformation. The temperature rising $\Delta T$ during plastic deformation can be calculated by the equation $\Delta T=\frac{\beta}{\rho C} \int_{0}^{\varepsilon_{f}} \sigma d \varepsilon$ [23], in which the conversion factor $\beta$ is usually taken as 0.9 (this implies that $10 \%$ of the deformation is stored in the material), $\rho$ is the density of Cu70-Zn30 $\left(8.52 \mathrm{~g} / \mathrm{cm}^{3}\right)$ and $C$ is the heat capacity $(385 \mathrm{~J} / \mathrm{kg} \cdot \mathrm{K})$. With the process of plastic strain, the number of twins and the twinned region in internal grain will increase. Large twins formed at the early stage will break because of severe plastic deformation, that leads to the thickness of average twin layer decrease. As continuous formation and growth of twins, the region which provides the space for later twin formation and growth will not form twins and gradually diminish. Then, the space forming twins reduced. Eventually, twins inside crystal will achieve saturated state when twin spacing and twin layer thickness reach the same size.

2.4. The Strain Rate Effect of Deformation Twinning. From Fig. 4, the twin spacing is decreasing with the increase of strain rate when the temperature $T$ increase from 77 up to $496 \mathrm{~K}$, respectively, but the strain rate effect is not obvious. Three different TB spacingstrain curves are nearly close under three different strain rate 1000,1 , and $0.001 \mathrm{~s}^{-1}$, it can be seen that the influence of strain rate on deformation twinning can be ignored. Comparing with Fig. $4 a$ and $4 b$, it is obvious that the influence of temperature on deformation twinning is much more sensitive to the influence of strain rate. The time of twin nucleation is mainly affected by the strain rate, for which high-stress concentration is also needed. The higher the strain rate, the faster the twin nucleation. Therefore, plastic deformation will be used to reduce the time of twin nucleation. On the other hand, that the higher the strain rate, the greater the number of twins, which can cause the reduction of the twin spacing. 


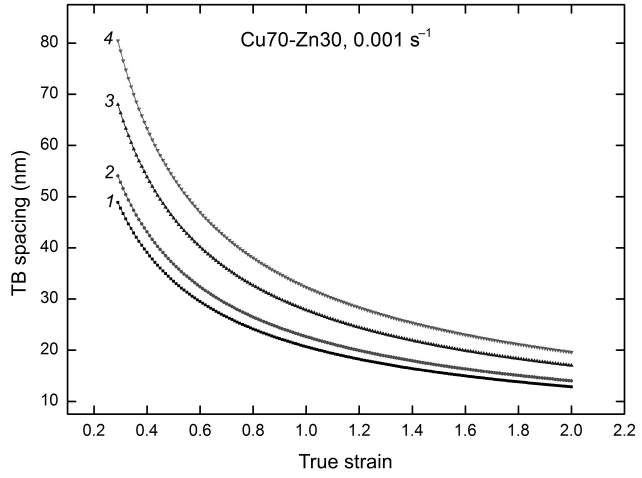

a

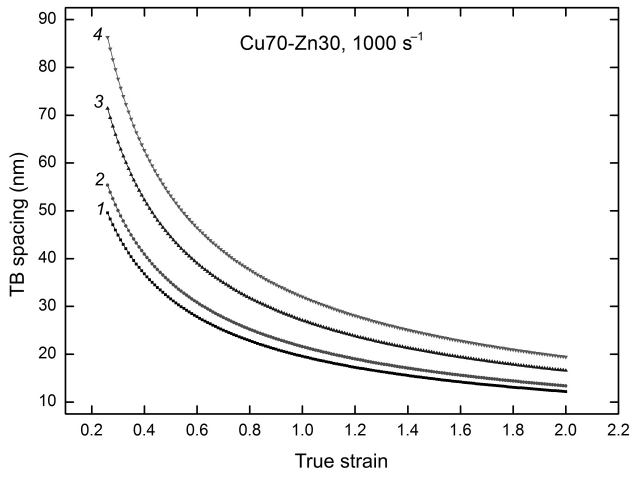

b

Fig. 3. The predictions for $\mathrm{Cu} 70-\mathrm{Zn} 30$ at 0.001 (a) and $1000 \mathrm{~s}^{-1}$ (b) strain rates and indicated temperature: (1) $T=77 \mathrm{~K}$, (2) $T=496 \mathrm{~K}$, (3) $T=896 \mathrm{~K}$, and (4) $T=1096 \mathrm{~K}$.

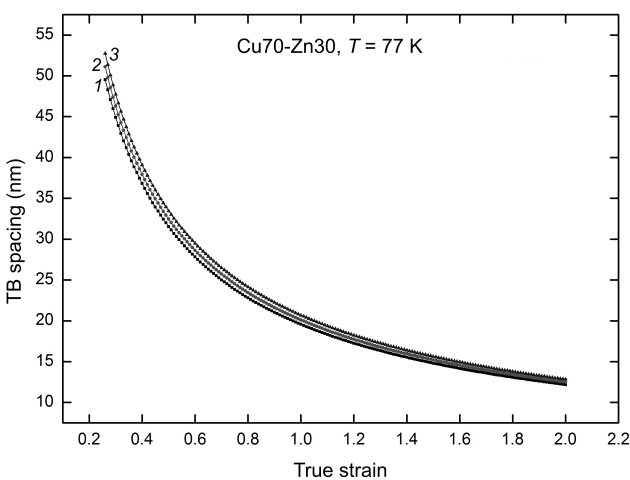

a

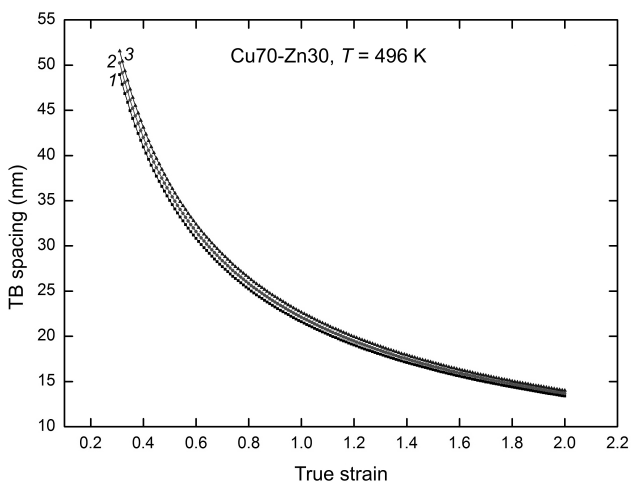

b

Fig. 4. TB spacing evolves with different strain rates at 77 (a) and $496 \mathrm{~K}$ (b): (1) $\dot{\varepsilon}=1000 \mathrm{~s}^{-1}$, (2) $\dot{\varepsilon}=1 \mathrm{~s}^{-1}$, and (3) $\dot{\varepsilon}=0.001 \mathrm{~s}^{-1}$.

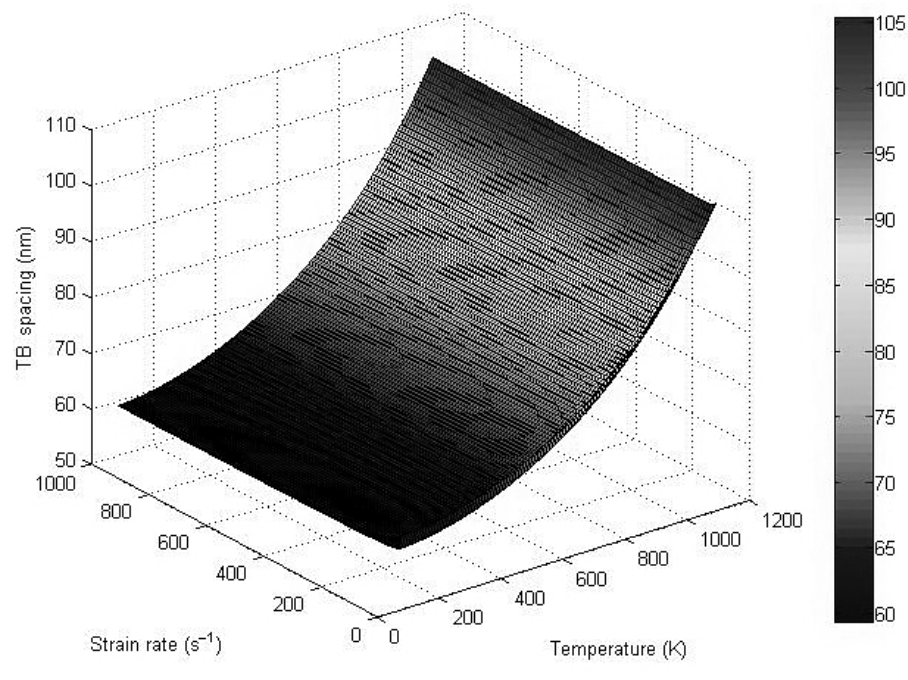



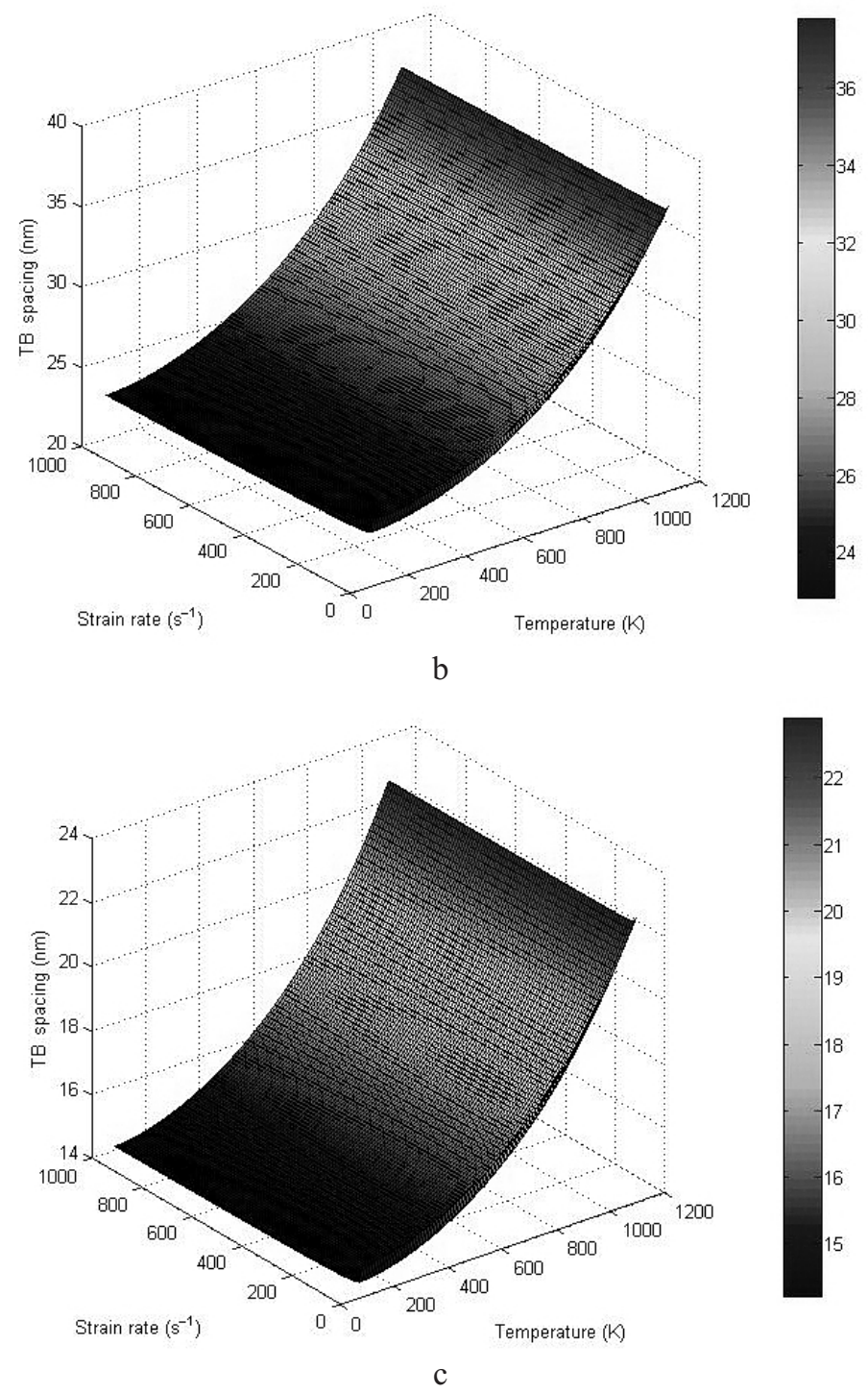

Fig. 5. Coupled effects of strain rates and temperature on deformation twinning when strain are 0.2 (a), 0.8 (b), and 1.6 (c).

\subsection{Couple Effects of Strain Rates and Temperature on Deformation Twinning.}

Figure 5 shows the couple effects of different strain rates and temperature on deformation twinning at the different strain. It is clear that the TB spacing decreases with the increase of strain rate and the decrease of temperature when the strain increase from 0.2 up to 1.6. Also, it can be seen that the influence of temperature on deformation twinning is much more sensitive than the influence of strain rate. In Fig. 5, when $T=1000 \mathrm{~K}, \dot{\varepsilon}=1000 \mathrm{~s}^{-1}$ the size of TB spacing reaches $14 \mathrm{~nm}$ that is close to the experimental results [16]. Combined with previous research, temperature and strain rate mainly affected the twin nucleation and the growth of twins. The higher the strain rate is, the faster the twin nucleation and the greater the number of twins.

Conclusions. In summary, a theoretical model which is used to validate the constitutive model is build to quantitatively analyze the deformation twinning of $\mathrm{Cu}-\mathrm{Zn}$ alloy, which 
considers the coupled effects of strain rate and temperature. In this research, $\mathrm{Cu} 70-\mathrm{Zn} 30$ owns a large number of twins at room temperature, and the twinning deformation which is affected by low temperature and high strain is the main deformation mechanism. It is well known that the thickness of twin layer will decrease when TB spacing decreases. When the deformation process begins, dislocations start to pile up against TBs and produce a stress concentration. So, the smaller the TB spacing, the larger the flow stress. From the theoretical model, it can be seen that keeping the strain rate is fixed, the TB spacing decreases with temperature decreasing. In the same way, when the temperature is not changed, the influence of strain rate on deformation twinning can be ignored, and the higher the strain rate, the faster the twin nucleation. As the different strain rates and temperature on deformation twinning at different strain, we know that temperature and strain rate mainly affected the twin nucleation and the growth of twins, and the influence of temperature on deformation twinning is much more sensitive to the influence of strain rate. Above all, the main conclusions can be drawn that low temperature and high strain rate will promote deformation twinning, and TB spacing will decrease with lower temperature and higher strain rate. However, the role of strain rate on deformation twinning in $\mathrm{Cu}-\mathrm{Zn}$ alloys is less obvious than the temperature.

Acknowledgments. This work was supported by National Natural Science Foundation of China (10872087, 11272143), Key Project of Chinese Ministry of Education (211061), Natural Science Foundation of Hubei Province (Q20111501) and the Program for Chinese New Century Excellent Talents in University (NCET-12-0712).

1. J. W. Christian and S. Mahajan, "Deformation twinning," Prog. Mater. Sci., 39, 1-157 (1995).

2. L. Zhu, H. Ruan, and X. Li, "Modeling grain size dependent optimal twin spacing for achieving ultimate high strength and related high ductility in nanotwinned metals," Acta Mater., 59, 5544-5557 (2011).

3. D. M. Kochmann and K. C. Le, "A continuum model for initiation and evolution of deformation twinning," J. Mech. Phys. Solids, 57, 987-1002 (2009).

4. A. P Stebner, S. C. Vogel, and R. D. Noebe, "Micromechanical quantification of elastic, twinning, and slip strain partitioning exhibited by polycrystalline, monoclinic nickel-titanium during large uniaxial deformations measured via in-situ neutron diffraction," J. Mech. Phys. Solids, 61, 2302-2330 (2013).

5. M. A. Meyers, O. Vohringer, and V. A. Lubarda, "The onset of twinning in metals: a constitutive description," Acta Mater., 49, 4025-4039 (2001).

6. L. Lu and K. Lu, "Ultrahigh strength and high electrical conductivity in copper," Science, 304, 422-426 (2004).

7. J. Schiøtz and K. W. Jacobsen, "A maximum in the strength of nanocrystalline copper," Science, 301, 1357-1359 (2003).

8. O. Johari and G. Thomas, "Substructures in explosively deformed $\mathrm{Cu}$ and $\mathrm{Cu}-\mathrm{Al}$ alloys," Acta Metall., 12, 1153-1159 (1964).

9. M. A. Crimp, B. C. Smith, and D. E. Mikkola, "Substructure development in shock-loaded $\mathrm{Cu}-8.7 \mathrm{Ge}$ and copper: the role of temperature, grain size and stacking fault energy," Mater. Sci. Eng., 96, 27-40 (1987).

10. L. E. Murr, E. V. Esquivel, and J. Mater, "Observations of common microstructural issues associated with dynamic deformation phenomena: twins, microbands, grain size effects, shear bands, and dynamic recrystallization," J. Mater. Sci., 39, 1153-1168 (2004). 
11. Y. M. Wang, T. Jiao, and E. Ma, "Dynamic processes for nanostructure development in $\mathrm{Cu}$ after severe cryogenic rolling deformation," Mater. Trans., 44, 1926-1934 (2003).

12. G. H. Xiao and N. R. Tao, "Effects of strain, strain rate and temperature on deformation twinning in a Cu-Zn alloy," Scripta Mater., 59, 975-978 (2008).

13. X. Liang and X. San, "Studies on the mechanism of the alloy compression behavior," J. Kunming Univ. Sci. Technol., 36, 23-28 (2011).

14. S. Nemat-Nasser and Y. Li, "Flow stress of fcc polycrystals with application to OFHC Cu," Acta Mater., 46, 565-577 (1998).

15. G. R. Johnson and W. H. Cook, "A constitutive model and data for metals subjected to large strains, high strain rates and high temperatures," in: Proc. of the Seventh Int. Symp. on Ballistics (April 19-21, 1983, the Hague, the Netherlands), the Hague (1983), pp. 541-547.

16. G. H. Xiao, N. R. Tao, and K. Lu, "Strength-ductility combination of nanostructured $\mathrm{Cu}-\mathrm{Zn}$ alloy with nanotwin bundles," Scripta Mater., 65, 119-122 (2011).

17. R. Kossowsky and N. Brown, "Microyielding in iron at low temperatures/ Microdeformation du fer a basse temperature/Mikroflie $\beta$ en in eisen bei tiefen temperaturen," Acta Metall., 14, 131-139 (1966).

18. M. A. Meyers and K. K. Chawla, Mechanical Behavior of Materials, Cambridge University Press (2009), ISBN: 978-0-521-86675-0.

19. S. Zhang, J. Zhou, L. Wang, and Y. Wang "The effect of the angle between loading axis and twin boundary on the mechanical behaviors of nanotwinned materials," Mater. Design, 45, 292-299 (2013).

20. R. J. Klassen and M. Haghshenas, "Indentation-based assessment of the dependence of geometrically necessary dislocations upon depth and strain rate in FCC materials," Mater. Sci. Eng. A, 586, 223-230 (2013).

21. M. Fisk, J. C. Ion, and L.-E. Lindgren, "Flow stress model for IN718 accounting for evolution of strengthening precipitates during thermal treatment," Comput. Mater. Sci., 82, 531-539 (2014).

22. I. Karaman, H. Sehitoglu, A. J. Beaudoin, et al., "Modeling the deformation behavior of Hadfield steel single and polycrystals due to twinning and slip," Acta Mater., 48, 2031-2047 (2000).

23. A. Mishra, B. K. Kad, F. Gregori, and M. A. Meyers, "Microstructural evolution in copper subjected to severe plastic deformation: experiments and analysis," Acta Mater., 55, 13-28 (2007). 